\title{
Characterization of a trifunctional fatty acid desaturase from oleaginous filamentous fungus Mortierella alpina 1S-4 using a yeast expression system.
}

\section{$\operatorname{AUTHOR}(S):$}

Kikukawa, Hiroshi; Sakuradani, Eiji; Kishino, Shigenobu; Park, Si-Bum; Ando, Akinori; Shima, Jun; Ochiai, Misa; Shimizu, Sakayu; Ogawa, Jun

\section{CITATION:}

Kikukawa, Hiroshi ...[et al]. Characterization of a trifunctional fatty acid desaturase from oleaginous filamentous fungus Mortierella alpina 1S-4 using a yeast expression system. Journal of bioscience and bioengineering 2013, 116(6): 672-676

\section{ISSUE DATE:}

2013-12

URL:

http://hdl.handle.net/2433/179615

\section{RIGHT:}

(C) 2013 The Society for Biotechnology, Japan. Published by Elsevier B.V.; この論文は出版社版でありません。引用の際には出版社版をご確 認ご利用ください。; This is not the published version. Please cite only the published version. 
1 Characterization of a trifunctional fatty acid desaturase from

2 oleaginous filamentous fungus Mortierella alpina 1S-4 using a yeast

expression system

Hiroshi Kikukawa $^{1}$, Eiji Sakuradani ${ }^{1 *}$, Shigenobu Kishino ${ }^{1}$, Si-Bum Park ${ }^{2}$, Akinori

6 Ando $^{3,4}$, Jun Shima ${ }^{4}$, Misa Ochiai ${ }^{5}$, Sakayu Shimizu ${ }^{6}$, and Jun Ogawa ${ }^{1,4}$

${ }^{1}$ Division of Applied Life Sciences, Graduate School of Agriculture, Kyoto University, Kitashirakawa-oiwakecho, Sakyo-ku, Kyoto 606-8502, Japan;

${ }^{2}$ Laboratory of Industrial Microbiology, Graduate School of Agriculture, Kyoto

${ }^{3}$ Research Unit for Physiological Chemistry, Kyoto University, 
$2 \quad{ }^{5}$ Institute for Microbial Science, Suntory Business Expert Ltd., 1-1-1

3 Wakayamadai, Shimamoto-cho, Mishima-gun, Osaka 618-8503, Japan;

$5 \quad{ }^{6}$ Faculty of Bioenvironmental Science, Kyoto Gakuen University, 1-1 Otani,

6 Nanjo, Sogabe-cho, Kameoka, Kyoto 621-8555, Japan

7

$8 \quad$ [Keywords; Mortierella alpina; Polyunsaturated fatty acid;

9 
A w3-fatty acid desaturase gene (maw3) which is involved in biosynthesis of

$2 n$-3 polyunsaturated fatty acids (PUFAs) was previously isolated from Mortierella

3 alpina 1S-4. In this report, we investigated the products of MAW3 catalyzing

4 reaction with endogenous and exogenous fatty acids in the yeast transformant.

5 Two unusual fatty acids de novo synthesized in the yeast transformant expressing

6 maw3 gene were identified as $n-4$ hexadecadienoic acid $\left(16: 2^{9 c i s, 12 c i s}\right)$ and $n-1$

7 hexadecatrienoic acid $\left(16: 3^{9 c i s, 12 c i s, 15}\right)$ by GC-MS and ${ }^{1}$ H-NMR analyses. In

8 addition to the desaturation activity at the $\omega 3$-position for 18- and 20-carbon

9 PUFAs, MAW3 in the yeast transformant inserted a double bond at $\Delta 12$-position of

10 endogenous palmitoleic acid $\left(16: 1^{9 c i s}\right)$ and further at $\Delta 15$-position of the resulting

$1116: 2^{9 c i s, 12 c i s}$ to result in the formation of $16: 3^{9 c i s, 12 c i s, 15}$ leading to a bifunctional

$12 \Delta \mathbf{\Delta 1 2} / \mathbf{\Delta 1 5}$-desaturase for 16-carbon fatty acids. Moreover, we evaluated the

13 activity of MAW3 in the yeast transformant under different temperatures. The

14 MAW3 did not have desaturation activities in $M$. alpina $1 S-4$ at $28^{\circ} \mathrm{C}$ but it had in

15 the yeast transformant for various fatty acids. The MAW3 was demonstrated to

16 be a trifunctional $\Delta 12 / \Delta 15 / \omega 3$-desaturase, exhibiting $\Delta 12$-desaturation for $16: 1^{9 c i s}$,

$17 \quad$ \$15-desaturation for 16- and 18-carbon fatty acids that had a preexisting

18 cis-double bond at $\mathbf{\Delta 1 2}$ position, and $\omega 3$-desaturation for 20 -carbon fatty acids 
1 having that at $\Delta 14$-position. It is the first report that the fatty acid desaturase

2 (MAW3) is shown to have $\mathbf{\Delta 1 2}$ - and $\mathbf{\Delta 1 5}$-desaturation activities for a 16 -carbon

3 fatty acid, in addition to its major function, $\omega 3$-desaturation activity. 
Biosynthetic pathways of fatty acids have been determined in many species by

2 identification of their genes encoding fatty acid desaturases (1-4). An oleaginous

3 filamentous fungus, Mortierella alpina $1 \mathrm{~S}-4$, has been shown to contain $\Delta 5-, \Delta 6-, \Delta 9-$,

$4 \Delta 12-$, and $\omega 3$-fatty acid desaturases and four kinds of elongases involved in

5 polyunsaturated fatty acids (PUFAs) biosynthesis (5). In $M$. alpina $1 \mathrm{~S}-4, \Delta 5-, \Delta 6-, \Delta 9-$,

6 and $\Delta 12$-desaturases exhibit their desaturation activity at a cultural temperature of $28^{\circ} \mathrm{C}$,

7 whereas $\omega 3$-fatty acid desaturase exhibits its activity only below a cultural temperature

8 of $20^{\circ} \mathrm{C}$.

M. alpina 1S-4 is capable of producing triacylglycerols rich in arachidonic acid

10 (ARA; $20: 4^{5 c i s, 8 c i s, 11 c i s, 14 c i s}$ ) with the amount ranging of 3-20 g/L of culture broth and

$1130-70 \%$ in the total fatty acid composition. On the other hand, this fungus

12 accumulates a small amount of eicosapentaenoic acid (EPA; 20:5 $5^{5 c i s, 8 c i s, 11 c i s, 14 c i s, 17 c i s}$ )

13 with $0.3 \mathrm{~g} / \mathrm{L}$ of culture broth and $10 \%$ in total fatty acids below a cultural temperature of

$1420^{\circ} \mathrm{C}(5)$. We had previously reported isolation of the gene (maw3) encoding an

15 (13-desaturase, involved in biosynthesis of $n$-3 PUFAs, from M. alpina $1 \mathrm{~S}-4$, and have

16 confirmed that the gene product (MAW3) converts $n$-6 PUFAs into $n-3$ PUFAs (6).

17 MAW3 possesses three histidine rich-cluster motifs containing eight histidine residues

18 that are proposed to form an active-site domain and two hydrophobic 
1 membrane-spanning regions in its deduced amino acid sequence $(6,7)$. Similar to $\Delta 12$ -

2 and $\omega 3$-desaturases found in other organisms, MAW3 has no cytochrome $b_{5}$ motif in its

3 structure $(6,8,9)$. Overexpression of maw3 gene in $M$. alpina $1 \mathrm{~S}-4$ through

4 Agrobacterium tumefaciens-mediated transformation led to an increase in EPA

5 production contributing to $40 \%$ of total fatty acid content (10).

6

Fatty acid desaturases are known to have five specificities: chemoselectivity

7 (desaturation or hydroxylation), regioselectivity (double bond position),

8 stereoselectivity (cis or trans), chain length specificity, and acyl carrier specificity

9 (acyl-CoA, acyl-lipid, or acyl-ACP substrate) (11). Three types of regioselectivities

10 have been defined (11). The $\Delta \mathrm{x}$-desaturases such as $\Delta 9$-desaturases insert a first

11 double bond at $\mathrm{x}$ carbon atom position away from the carboxyl end. On the other hand,

12 the $\omega y$-desaturases such as $\omega 3$-desaturases insert a double bond at position y from the

13 methyl end. The $\mathrm{v}+\mathrm{z}$-desaturases require a preexisting double bond as a mark ( $\mathrm{v})$ and

14 catalyze the formation of a new double bond $\mathrm{z}$ carbon atoms further along the acyl chain,

15 either toward the methyl end (i.e. $\Delta 12$-desaturase) or toward the carboxyl end (i.e.

$16 \Delta 6$-desaturase). The aforementioned types of regioselectivity are explained for

17 bifunctional fungal $\Delta 12 / \omega 3$-desaturases from Fusarium moniliforme (12) and

18 Aspergillus nidulans (13). These bifunctional enzymes desaturate oleic acid (OA; 
$1 \quad 18: 1^{9 c i s}$ ) at $\Delta 12$-position to form linoleic acid (LA; 18:2 $2^{9 c i s, 12 c i s}$ ) and further at the

$2 \Delta$ 15-position to form $\alpha$-linolenic acid (ALA; 18:3 ${ }^{9 c i s, 12 c i s, 15 c i s}$ ). Additionally, these

3 desaturases also convert $n$-6 PUFAs: LA, $\gamma$-linolenic acid (GLA; 18:3 $3^{6 c i s, 9 c i s, 12 c i s}$ ),

4 dihomo- $\gamma$-linolenic acid (DGLA; 20:3 $3^{8 c i s, 11 c i s, 14 c i s}$ ), and ARA to their corresponding $n$-3

5 PUFAs with preference of the 18-carbon PUFAs. Other bifunctional

$6 \Delta 12 / \Delta 15$-desaturases that catalyze the synthesis of unusual $n-1$ hexadecatrienoic acid

$7 \quad\left(16: 3^{9 c i s, 12 c i s, 15}\right)$ have been reported in the free-living soil protozoon Acanthamoeba

8 castellanii (14), phytopathogenic fungus Claviceps purpurea (15), basidiomycete

$9 \quad$ Coprinus cinereus (16), and nematode Caenorhabditis elegans (17).

In this study, we attempted to characterize the MAW3 using the yeast

11 Saccharomyces cerevisiae expression system. Two unusual fatty acids synthesized de novo in maw3-expressing yeast transformant were identified by GC-MS and NMR. The MAW3 in yeast transformant inserted a double bond at $\Delta 12$-position and further at

$14 \Delta 15$-position for endogenous 16 -carbon fatty acids as well as at $\omega 3$-position of exogenous 18- and 20-carbon PUFAs. The M. alpina 1S-4 $\omega 3$-desaturase was found to

16 be a trifunctional $\Delta 12 / \Delta 15 / \omega 3$-desaturase that mainly acts as $\Delta 15$ - and $\omega 3$-desaturase,

17 and as a $\Delta 12$-desaturase for 18- and 20-carbon PUFAs in addition to 16-carbon fatty

18 acids. The finding is different from other bifunctional desaturases so far reported 
$1 \quad(12-17)$. 
Strains, plasmid, growth media, and culture conditions The maw3 gene from

M. alpina 1S-4 (18) was registered in the DDBJ database under the accession number

AB182163 (6). S. cerevisiae EH1315 ( $\alpha$ trpl) was used as the recipient strain in transformation experiments (19). Plasmid pYE22m, used as a shuttle vector, carried the ampicillin resistance gene (for selection in Escherichia coli) and TRP1 (for tryptophan prototrophy selection in S. cerevisiae EH1315), with an expression of a target gene controlled under the glyceraldehyde-3-phosphate dehydrogenase (GAP) promoter and the GAP terminator from $S$. cerevisiae.

M. alpina $1 \mathrm{~S}-4$ strain was cultured in GY medium (containing $20 \mathrm{~g} / \mathrm{L}$ of glucose, $10 \mathrm{~g} / \mathrm{L}$ of yeast extract, $\mathrm{pH} 6.0$ ) for 5 days at $28^{\circ} \mathrm{C}$. Yeast strains were cultured in a yeast synthetic complete medium (SC medium; containing $20 \mathrm{~g} / \mathrm{L}$ of glucose, $5 \mathrm{~g} / \mathrm{L}$ of ammonium sulfate, $1.7 \mathrm{~g} / \mathrm{L}$ of yeast nitrogen base without amino acids and ammonium sulfate, $60 \mathrm{mg} / \mathrm{L}$ of isoleucine, $60 \mathrm{mg} / \mathrm{L}$ of leucine, $60 \mathrm{mg} / \mathrm{L}$ of phenylalanine, $50 \mathrm{mg} / \mathrm{L}$ of threonine, $40 \mathrm{mg} / \mathrm{L}$ of lysine, $30 \mathrm{mg} / \mathrm{L}$ of tyrosine, $20 \mathrm{mg} / \mathrm{L}$ of adenine, $20 \mathrm{mg} / \mathrm{L}$ of arginine, $20 \mathrm{mg} / \mathrm{L}$ of uracil, $20 \mathrm{mg} / \mathrm{L}$ of histidine, and $10 \mathrm{mg} / \mathrm{L}$ of methionine) for 2 days at $15^{\circ} \mathrm{C}$ (or at $\left.28^{\circ} \mathrm{C}\right)$. 
1 a total reaction volume of $50 \mu$ l sample containing $<0.2 \mu \mathrm{g}$ of plasmid pYMAW3 (6) as

2 template DNA, $0.5 \mu \mathrm{l}$ of PrimeSTAR HS DNA polymerase (Takara Bio, Shiga, Japan),

$310 \mu \mathrm{l}$ of $5 \times$ PrimeSTAR buffer $\left(\mathrm{Mg}^{2+}\right.$ plus $), 200 \mu \mathrm{M}$ of each dNTP and $200 \mathrm{pM}$ of each

4 primer: $\omega 3$-1F (5'-ATGGCCCCCCCTCACGTTGTCGACGAACA-3') containing an

5 ATG start site (underline) and

6 (5'-TTAATGCTTGTAAAACACTACATCTCC-3') containing a TAA stop site

7 (underline). PCR amplification was performed as follows: initial denaturation at $94^{\circ} \mathrm{C}$

8 for $2 \mathrm{~min}$, followed by 25 cycles of $98^{\circ} \mathrm{C}$ for $10 \mathrm{sec}, 55^{\circ} \mathrm{C}$ for $15 \mathrm{sec}$, and $72^{\circ} \mathrm{C}$ for 2

9 min. The resultant 1212-bp PCR fragment was digested with EcoRI and treated with

DNA Blunting Kit (Takara Bio) for blunt-end ligation into the yeast expression vector

11 pYE22m to result in the construction of a plasmid, designated as pYE22MAW3, which

was further transformed into the S. cerevisiae EH1315 strain by means of the lithium acetate-mediated transformation protocol (20). Transformants was selected for tryptophan (Trp) auxotrophy on SC (-Trp) medium. medium. To test for the substrate specificities of MAW3 in yeast, various free fatty acids (FFAs) were added to the culture broth at $0.1 \mathrm{mM}$ concentration after $24 \mathrm{~h}$ of cultivation. The yeast transformant with an empty vector was used as the control 
1 strain.

2

Fatty acid analysis Total fatty acid compositions of the yeast transformants were analyzed as described by Sakuradani et al. (6). Yeast cells after cultivation were harvested by centrifugation and dried at $120^{\circ} \mathrm{C}$ for $1.5 \mathrm{~h}$. The dried cells were directly transmethylated with $10 \%$ methanolic $\mathrm{HCl}$ at $55^{\circ} \mathrm{C}$ for $1.5 \mathrm{~h}$. The resultant fatty acid methyl esters (FAMEs) were extracted with $n$-hexane, concentrated and analyzed by gas chromatography (GC; Shimadzu, Kyoto, Japan). The conversion rate of substrate into the product was calculated as the enzymatic activity of the desaturase [conversion rate $(\%)=100 \times \operatorname{product} /($ product + substrate $)]$.

Total lipids were extracted from wet yeast cells using water/chloroform/methanol $(2 / 2.5 / 2.5, \mathrm{v} / \mathrm{v} / \mathrm{v})$ according to the Bligh-Dyer method (21). The extracted lipids were saponified to obtain their FFAs using $0.5 \mathrm{~N} \mathrm{KOH}$ in $90 \% \mathrm{MeOH}$. The unidentified FFAs were separated by HPLC with a LC-20A system (Shimadzu). A Develosil C30-UG-5 column $(4.6 \times 250 \mathrm{~mm}$, Nomura chemical, Aichi, Japan) was used for FFA separation by HPLC with $80 \%$ (v/v) acetonitrile aqueous solution as a mobile phase at a flow rate of $1.0 \mathrm{ml} / \mathrm{min}$. The column oven temperature was maintained at $35^{\circ} \mathrm{C}$, and the absorbance was measured by UV/VIS detector at $205 \mathrm{~nm}$. The mixture of FFAs separated by HPLC were transmethylated with methanol/benzene/1\% diazomethane 
$1 \quad(4 / 6 / 1, \mathrm{v} / \mathrm{v} / \mathrm{v})$ for $30 \mathrm{~min}$ at room temperature for preparation of FAMEs. Through the

2 Discovery Ag-Ion SPE column (SUPELCO, Bellefonte, PA, USA), most of FAMEs in

3 the UK2 fraction except for the UK2 were removed with acetone and the UK2 was

4 eluted with acetonitrile. The 4,4-Dimethyloxazoline (DMOX) derivatives of the UK1

5 and UK2 obtained through the HPLC purification was prepared and analyzed by

6 GC-MS as described by Yu et al. (22). A GC-MS QP2010 (Shimadzu) with GC-2010

7 gas chromatograph was used for MS analysis. The $\mathrm{SPB}^{\mathrm{TM}-1}$ column $(0.25 \mathrm{~mm}$ I.D. $\times$

$830 \mathrm{~m}$, SUPELCO) was used for separation of DMOX derivatives. Initial column

9 temperature was set at $180^{\circ} \mathrm{C}$ and was raised to $300^{\circ} \mathrm{C}$ later at a rate of $5^{\circ} \mathrm{C} / \mathrm{min}$. The apparatus provides the electron impact mode at $70 \mathrm{eV}$ with a source temperature of $250^{\circ} \mathrm{C}$. The injection temperature was $250^{\circ} \mathrm{C}$. The ${ }^{1} \mathrm{H}-\mathrm{NMR}$ and DQF-COSY experiments were performed with a Bruker Advance $500(500 \mathrm{MHz})$. Samples were dissolved in chloroform-d (Sigma, St. Louis, MO, USA). Chemical shifts were assigned relative to the solvent signal.

\section{RESULTS}


1 characterize the maw3 gene product from M. alpina $1 \mathrm{~S}-4$ by using a yeast expression

2 system, the yeast transformant with pYE22MAW3 containing the maw3 cDNA was

3 obtained. The yeast transformants were grown in SC medium, and their total fatty acid

4 compositions were analyzed by GC (Fig. 1A and 1B). The maw3-expressing yeast

5 transformant accumulated two unusual fatty acids, named UK1 and UK2 respectively,

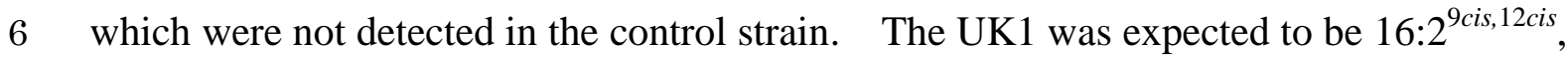

7 because the retention time of the UK1 was consistent with that of an authentic standard

$8 \quad$ (Fig. 1C).

Each FFA of UK1 and UK2 was purified by HPLC equipped with a Develosil

C30-UG-5 column, followed by the further purification through Discovery Ag-Ion SPE

11 column for UK2. The positions of the double bonds in UK1 and UK2 were determined by GC-MS analysis of their DMOX derivatives (Fig. 2). MS analysis of UK1 and UK2 DMOX derivatives in Fig. 2 revealed molecular ions of $\mathrm{m} / z, 305$ and 303, and the gaps of 26 atomic mass unit (amu) between $\mathrm{m} / z, 196$ and 222, and $\mathrm{m} / z 236$ and 262 , indicating double bond at the $\Delta 9$ - and $\Delta 12$-positions in their structures, respectively.

16 The gap of 41 amu between $m / z, 262$ and 303 in the mass spectrum of UK2 (Fig. 2B)

17 suggested a double bond at the $\Delta 14$ - or $\Delta 15$-position. To determine a position of a 18 double bond at methyl terminal of UK2 completely, the FAME of UK2 showing 95\% 
1 purity on GC chromatogram was applied for ${ }^{1} \mathrm{H}-\mathrm{NMR}$ analysis (Fig. 3) and DQF-COSY

2 analysis (FIGURE S1). ${ }^{1} \mathrm{H}-\mathrm{NMR} \delta_{\mathrm{H}}\left(\mathrm{CDCl}_{3}\right)$ spectral data of UK2: $5.82(1 \mathrm{H}, t t$,

$\left.3-\mathrm{CH}_{2}-\mathrm{CH}=\mathrm{CH}_{2}, \mathrm{H}-15\right), 5.38\left(4 \mathrm{H}, m,-\mathrm{CH}_{2}-\mathrm{CH}=\mathrm{CH}-, \mathrm{H}-9, \mathrm{H}-10, \mathrm{H}-12, \mathrm{H}-13\right), 5.02(2 \mathrm{H}$,

$\left.4 d, J=10.1,17.0 \mathrm{~Hz},-\mathrm{CH}_{2}-\mathrm{CH}=\mathrm{CH}_{2}, \mathrm{H}-16\right), 3.67\left(3 \mathrm{H}, s,-\mathrm{C}(=\mathrm{O})-\mathrm{O}-\mathrm{CH}_{3}\right), 2.83(2 \mathrm{H}, d d$,

$\left.5 J=6.07,6.07 \mathrm{~Hz},=\mathrm{CH}-\mathrm{CH}_{2}-\mathrm{CH}=, \mathrm{H}-14\right), 2.79(2 \mathrm{H}, d d, \quad J=6.07,6.07 \mathrm{~Hz}$,

$\left.6=\mathrm{CH}-\mathrm{CH}_{2}-\mathrm{CH}=, \mathrm{H}-11\right), 2.30\left(2 \mathrm{H}, t, J=7.28 \mathrm{~Hz},-\mathrm{C}(=\mathrm{O})-\mathrm{CH}_{2}-\mathrm{CH}_{2^{-}}, \mathrm{H}-2\right), 2.05(2 \mathrm{H}, d t$,

$\left.7 J=6.78,6.78 \mathrm{~Hz}, \quad-\mathrm{CH}_{2}-\mathrm{CH}_{2}-\mathrm{CH}=, \mathrm{H}-8\right), 1.679(2 \mathrm{H}, \quad t t, \quad J=7.33,7.41 \mathrm{~Hz}$,

$\left.8-\mathrm{CH}_{2}-\mathrm{CH}_{2}-\mathrm{CH}_{2^{-}}, \mathrm{H}-3\right)$, and $1.31\left(8 \mathrm{H}, m,-\mathrm{CH}_{2}-\mathrm{CH}_{2}-\mathrm{CH}_{2^{-}}, \mathrm{H}-4, \mathrm{H}-5, \mathrm{H}-6, \mathrm{H}-7\right)$. The

9 absorption peaks at $\delta 5.82\left(-\mathrm{CH}_{2}-\mathrm{CH}=\mathrm{CH}_{2}, \mathrm{H}-15\right)$ and $5.02\left(-\mathrm{CH}_{2}-\mathrm{CH}=\mathrm{CH}_{2}, \mathrm{H}-16\right)$ in

10 Fig. 3 revealed a double bond at the $\Delta 15$-position in the structure of UK2. From these

11 analyses, UK1 and UK2 were identified as $16: 2^{9 c i s, 12 c i s}$ and $16: 3^{9 c i s, 12 c i s, 15}$, respectively.

13 transformant was different from that of the control strain. The $16: 2^{9 c i s, 12 c i s}$ and

$1416: 3^{9 c i s, 12 c i s, 15}$ comprised $0.8 \%$ and $4.0 \%$ of the total fatty acid content, respectively,

15 which were not detected in the control strain. The percentage of $16: 1^{9 c i s}$ was decreased

16 slightly in the yeast transformant, compared to that in the control strain. Neither LA

17 nor ALA was detected in both yeast strains, suggesting that MAW3 in the transformant

18 prefers a 16-carbon fatty acid like $16: 1^{9 c i s}$ to an 18 -carbon fatty acid like OA. 
1

2

3

$4 \quad 18: 1^{9 c i s}$ (OA), crepenynic acid (9cis-octadecen-12-ynoic acid; 18:19cis,12yn $)$,

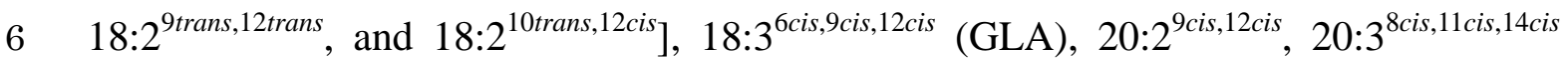

7 (DGLA), and 20:4 ${ }^{5 c i s, 8 c i s, 11 c i s, 14 c i s}$ (ARA). Conversions of the substrates by the

8 maw3-expressing yeast transformant were carried out during cultivation at $15^{\circ} \mathrm{C}$ or

$928^{\circ} \mathrm{C}$ (Table 2). M. alpina $1 \mathrm{~S}-4$ has no $\omega 3$-desaturation activity at $28^{\circ} \mathrm{C}$ cultivation,

10 whereas the yeast transformant exhibited the activity for the substrates even at $28^{\circ} \mathrm{C}$.

11 The MAW3 in the yeast transformant desaturated the following PUFAs: $16: 2^{9 c i s, 12 c i s}$,

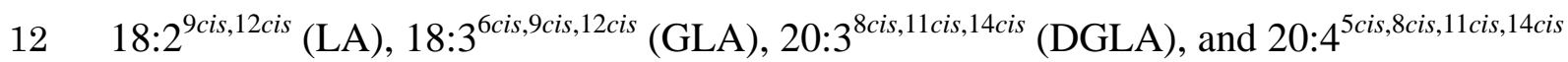

13 (ARA) to $16: 3^{9 c i s, 12 c i s, 15}, 18: 3^{9 c i s, 12 c i s, 15 c i s}$ (ALA), stearidonic aicd (SDA;

$\left.14 \quad 18: 4^{6 c i s, 9 c i s, 12 c i s, 15 c i s}\right), \quad$ eicosatetraenoic $\quad$ acid $\quad\left(\mathrm{ETA} ; \quad 20: 4^{8 c i s, 11 c i s, 14 c i s, 17 c i s}\right), \quad$ and

20:5 $5^{5 c i s, 8 c i s, 11 c i s, 14 c i s, 17 c i s}$ (EPA). Putative $\Delta 15$-desaturation products of $18: 2^{9 c i s, 12 y n, 15 c i s}$

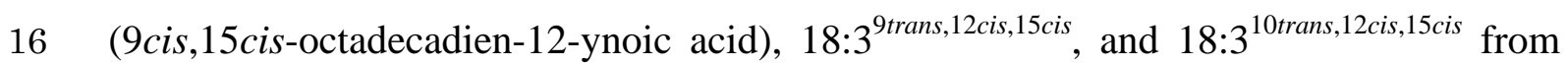

$1718: 1^{9 c i s, 12 y n}, 18: 2^{9 \text { trans, } 12 c i s}, 18: 2^{10 \text { trans, } 12 c i s}$, respectively, were also observed on GC

18 chromatograms. The $n-6$ PUFAs (LA, GLA, DGLA, and ARA) were more effectively 
1 converted into $n$-3 PUFAs (ALA, SDA, ETA, and EPA) at a low temperature $\left(15^{\circ} \mathrm{C}\right.$ )

2 cultivation of $M$. alpina $1 \mathrm{~S}-4$ than at $28^{\circ} \mathrm{C}$ temperature. In addition, conversion rates

3 for $16: 2^{9 c i s, 12 c i s}$ and crepenynic acid were observed at similar or higher levels at $28^{\circ} \mathrm{C}$ as

4 compared to the ones at $15^{\circ} \mathrm{C}$. Neither $\Delta 15$-desaturation nor $\omega 3$-desaturation products

5 were detected for $20: 2^{9 c i s, 12 c i s}$ in the yeast transformant.

6

The $\Delta 12$-desaturation catalyzed by MAW3 was observed only for $16: 1^{9 c i s}$, and

7 not for $18: 1^{9 c i s}$ and $18: 2^{6 c i s, 9 c i s}$. The high conversion rate with more than $60 \%$ was

8 shown for $16: 2^{9 c i s, 12 c i s}$ to $16: 3^{9 c i s, 12 c i s, 15}$ by $\Delta 15$-desaturation of MAW3 in the yeast

9 transformant. Furthermore, the $\omega 3$-desaturation of MAW3 was observed for 18- and

10 20-carbon PUFAs having a preexisting 12cis-double bond or 12-triple bond and a

11 preexisting 14cis-double bond, respectively.

\section{DISCUSSION} in ARA, but also accumulates $n$-3 PUFAs such as EPA below a cultural temperature of 
1 and characterized as to its function of $n-6$ PUFAs conversion to $n-3$ PUFAs in $M$. alpina

$2 \quad 1 \mathrm{~S}-4(6)$

In this study, the maw3-expressing yeast transformant was found to accumulate

4 two newly synthesized fatty acids, $16: 2^{9 c i s, 12 c i s}$ and $16: 3^{9 c i s, 12 c i s, 15}$. Thus far, we had no

5 idea that the MAW3 had $\Delta 12$-deasturase activity as an alternative function. The

6 accumulation of $16: 2^{9 c i s, 12 c i s}$ and $16: 3^{9 c i s, 12 c i s, 15}$ suggested that the yeast transformant had

7 both $\Delta 12$-desaturation activity with a conversion rate of $10.2 \%$ (calculated as $100 \times$

$8 \quad[16: 2+16: 3] /[16: 1+16: 2+16: 3])$ for endogenous $16: 1^{9 c i s}$, and $\Delta 15$-desaturation one at

$9 \omega 1$-position, not $\omega 3$, with a conversion rate of $83.0 \%$ (calculated as $100 \times[16: 3] /[16: 2+$

$1016: 3]$ ) for the resultant $16: 2^{9 c i s, 12 c i s}$. Once the $16: 2^{9 c i s, 12 c i s}$ was formed from $16: 1^{9 c i s}$ by

$11 \Delta$ 12-deasturation, $16: 2^{9 c i s, 12 c i s}$ was demonstrated to be rapidly converted to

$1216: 3^{9 c i s, 12 c i s, 15}$.

had w3-desaturation activity for 18- and 20-carbon PUFAs, but also demonstrated bifunctional $\Delta 12 / \Delta 15$-desaturation activities for 16 -carbon fatty acids. The $\Delta 12-, \Delta 15-$,

16 or $\omega 3$-desaturation activity of MAW3 requires a preexisting double bond at $\Delta 9-, \Delta 12-$,

17 or $\omega 6$-position, respectively. Hence, MAW3 is classified as $\mathrm{v}+3$ desaturase (v

18 indicating the position of the preexisting double bond) which inserted a new double 
1 bond 3 carbon atoms further along the acyl chain toward the methyl end.

basidiomycete $C$. cinereus (16); nematode $C$. elegans (17)], MAW3 in the yeast

4 transformant exhibited $\Delta 12$-desaturation for $16: 1^{9 c i s}$, not $18: 1^{9 c i s}$, and in addition to that,

5 also exhibited $\Delta 15$-desaturation for $16: 2^{9 c i s, 12 c i s}$ with a high conversion rate $(83.0 \%)$.

6 The previously reported bifunctional $\Delta 12 / \Delta 15$-desaturases mainly act as a

$7 \quad \Delta 12$-desaturase, except for the fungal $\omega 3$-desaturases described by Damude et al. (12)

8 and Hoffmann et al. (13), however do not possess $\Delta 12$-desaturation activity for the

9 16-carbon fatty acid. The ratios of $\omega 3 / \omega 6$ fatty acids (calculated as $[16: 3+18: 3] /[16: 2$

$10+18: 2$ ]) in yeast transformant were calculated as follows: 5.0 [MAW3, described here],

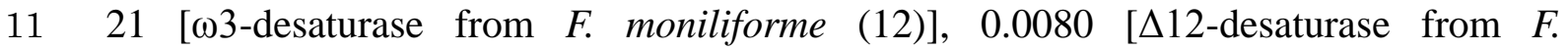

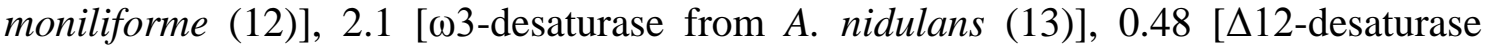
from A. castellanii (14)], 0.042 [ $\Delta 12$-desaturase from C. cinereus (16)], and 0.090

14 [ $\Delta 12$-desaturase from $C$. elegans $(17)]$ respectively based on the information derived

15 from each report. The bifunctional $\Delta 12 / \Delta 15$-desaturases from $F$. moniliforme and that

16 has a high ratio of 21 , mainly work as a $\omega 3$-desaturase, which prefers the 18 -carbon

17 PUFAs as substrates and not the 16-carbon fatty acids. On the other hand, the

18 bifunctional $\Delta 12 / \Delta 15$-desaturase from $C$. purpurea (15) introduces a double bond for 
1 both 16- and 18-carbon fatty acids, demonstrating increased activity of $\Delta 12$-desaturation

2 as compared to $\Delta 15$-desaturation.

On investigation of substrate specificities, the characteristic feature of MAW3 was shown to have both the $\Delta 12 / \Delta 15$-desaturation activity for the 16 -carbon fatty acids and $\omega 3$-desaturation activity for 18- and 20-carbon PUFAs, indicating its substrate specificities that differed from the other bifunctional $\Delta 12 / \Delta 15$-desaturases (12-17). It

7 is of interest to note that, the maw3-expressing yeast transformant exhibited the $\omega 3$-desaturation activity on cultivation at $28^{\circ} \mathrm{C}$, whereas $M$. alpina $1 \mathrm{~S}-4$ had no such w3-deaturation activity under similar culture conditions. This may imply the possibility of some regulation in the translation process and/or localization of MAW3 in M. alpina 1S-4 in vivo. The localization of MAW3 might be detected in cells of $M$. alpina $1 \mathrm{~S}-4$ by means of labeling techniques. We expect that the elucidation of the mechanism for the difference in MAW3 activity (depending on the culture temperature) may contribute to the accumulation of basic information on membrane-bound desaturases and to efficient production of n-3 PUFAs by $M$. alpina breeding.

\section{ACKNOWLEDGMENTS}


1 (no. 22380051 to E.S. and no. 23248014 to J.O.), the Program for Promotion of Basic

2 and Applied Researches for Innovations in Bio-oriented Industry of Japan, and

3 Advanced Low Carbon Technology Research and Development Program of Japan.

\section{References}

6

7 1. Napier, J. A., Michaelson, L. V.: Genomic and functional characterization of polyunsaturated fatty acid biosynthesis in Caenorhabditis elegans, Lipids, 36:761-766 (2001)

2. Uttaro, A. D.: Biosynthesis of polyunsaturated fatty acids in lower eukaryotes, IUBMB Life, 58:563-571 (2006).

3. Sakuradani, E., Ando, A., Ogawa, J., Shimizu, S.: Improved production of various polyunsaturated fatty acids through filamentous fungus Mortierella alpina breeding, Appl. Microbiol. Biotechnol., 84:1-10 (2009). acid desaturases from animals and lower eukaryotes, Prostaglandins Leukot. Essent. Fatty Acids, 68:97-106 (2003).

5. Sakuradani, E.: Advances in the Production of Various Polyunsaturated Fatty 
1

2

Acids through Oleaginous Fungus Mortierella alpina Breeding, Biosci. Biotechnol.

Biochem., 74: 908-917 (2010).

6. Sakuradani, E., Abe, T., Iguchi, K., and Shimizu, S.: A novel fungal

$\omega 3$-desaturase with wide substrate specificity from arachidonic acid-producing Mortierella alpina 1S-4, Appl. Microbiol. Biotechnol., 66: 648-654 (2005).

7. Los, D. A., Murata, N.: Structure and expression of fatty acid desaturases, Biochim. Biophys. Acta., 1394:3-15 (1998).

8. Petrini G. A., Altabe S. G., Uttaro A. D.: Trypanosoma brucei oleate desaturase may use a cytochrome $b 5$-like domain in another desaturase as an electron donor, Eur. J. Biochem., 271:1079-1086 (2004).

\section{Yazawa, H., Iwahashi, H., Kamisaka, Y., Kimura, K., Uemura, H.:}

Improvement of polyunsaturated fatty acids synthesis by the coexpression of $C Y B 5$ with desaturase genes in Saccharomyces cerevisiae, Appl. Microbiol. Biotechnol., 87:2185-2193 (2010).

10. Ando, A., Sumida, Y., Negoro, H., Suroto, D. A., Ogawa, J., Sakuradani, E., and Shimizu, S.: Establishment of Agrobacterium tumefaciens-mediated transformation of an oleaginous fungus, Mortierella alpina 1S-4, and its application for eicosapentaenoic acid producer breeding, Appl. Environ. Microbiol., 75: 5529-5535 
1

(2009)

11. Meesapyodsuk, D., Reed, D. W., Savile, C. K., Buist, P. H., Ambrose, S. J., and Covello, P. S.: Characterization of the Regiochemistry and Cryptoregiochemistry of a Caenorhabditis elegans Fatty Acid Desaturase (FAT-1) Expressed in Saccharomyces cerevisiae, Biochemistry, 39: 11948-11954 (2000).

12. Damude, H. G., Zhang, H., Farrall, L., Ripp, K. G., Tomb, J. F., Hollerbach, D., and Yadav, N. S.: Identification of bifunctional $\Delta 12 / \omega 3$ fatty acid desaturases for improving the ratio of $\omega 3$ to $\omega 6$ fatty acids in microbes and plants, Proc. Natl. Acad. Sci. U S A, 103: 9446-9451 (2006).

13. Hoffmann, M., Hornung, E., Busch, S., Kassner, N., Ternes, P., Braus, G. H., and Feussner, I.: A Small Membrane-peripheral Region Close to the Active Center Determines Regioselectivity of Membrane-bound Fatty Acid Desaturases from Aspergillus nidulans, J. Biol. Chem., 282: 26666-26674 (2007).

14. Sayanova, O., Haslam, R., Guschina, I., Lloyd, D., Christie, W. W., Harwood, J. L., and Napier, J. A.: A bifunctional $\Delta 12, \Delta 15$-desaturase from Acanthamoeba castellanii directs the synthesis of highly unusual $n-1$ series unsaturated fatty acids, J. Biol. Chem., 281: 36533-36541 (2006).

15. Meesapyodsuk, D., Reed, D. W., Covello, P. S., and Qiu, X.: Primary Structure, 
Regioselectivity, and Evolution of the Membrane-bound Fatty Acid Desaturases of Claviceps purpurea, J. Biol. Chem., 282: 20191-20199 (2007).

16. Zhang, S., Sakuradani, E., Ito, K., and Shimizu, S.: Identification of a novel bifunctional $\Delta 12 / \Delta 15$ fatty acid desaturase from a basidiomycete, Coprinus cinereus TD\#822-2, FEBS Lett., 581: 315-319 (2007).

17. Zhou, X. R., Green, A. G., and Singh, S. P.: Caenorhabditis elegans $\Delta 12$-desaturase FAT-2 is a bifunctional desaturase able to desaturate a diverse range of fatty acid substrates at the $\Delta 12$ and $\Delta 15$ positions, J. Biol. Chem., 286: 43644-43650 (2011).

18. Yamada, H., Shimizu, S., and Shinmen, Y.: Production of arachidonic acid by Mortierella elongata 1S-5, Agric. Biol. Chem., 51: 785-790 (1987).

19. Ashikari, T., Kiuchi-Goto, N., Tanaka, Y., Shibano, Y., Adachi, T., and Yoshizumi, H.: High expression and efficient secretion of Rhizopus oryzae glucoamylase in the yeast Saccharomyces cerevisiae, Appl. Microbiol. Biotechnol., 30: $515-520$ (1989).

20. Ito, H., Fukuda, Y., Murata, K., and Kimura, A.: Transformation of intact cells treated with alkali cations, J. Bacteriol., 153:163-168 (1983).

21. Bligh, E. G., and Dyer, W. J.: A rapid method of total lipid extraction and 
$1 \quad$ purification, Can. J. biochem physiol., 37: 911-917 (1959).

2 22. Yu, Q. T., Liu, B. N., Zhang, J. Y., and Huang, Z. H.: Locationof amrthyl branchings in fatty acids: Fatty acids in uropygial secretion of Shanghai duck by GC-MS of 4,4-dimethyloxazoline derivatives, Lipids, 8: 33-44 (1988).

23. Shimizu, S., Kawashima, H., Shinmen, Y., Akimoto, K., and Yamada, H.: Production of eicosapentaenoic acid by Mortierella fungi, J. Am. Oil. Chem. Soc., 65: $1455-1459$ (1988). 


\section{Figure legends}

2

3 FIG. 1. GC chromatograms of FAMEs prepared from total lipids of the control strain

4 (pYE22m) (A), the maw3-expressing yeast transformant (pYE22MAW3) (B), and the $5 \quad$ FAMEs of $16: 2^{9 c i s, 12 c i s}(\mathrm{C})$.

6

7 FIG. 2. Mass spectra of DMOX derivatives of (A) UK1 and (B) UK2 obtained from the

8 yeast transformant (pYE22MAW3). The specific mass fragments of authentic

$916: 2^{9 c i s, 12 c i s}$ were $m / z, 113,126,140,154,168,182,196,208,222,236,248,262,276$,

$10 \quad 276,290$, and 305.

11

12 FIG. 3. ${ }^{1} \mathrm{H}-\mathrm{NMR}$ analysis of the UK2 methyl ester. 

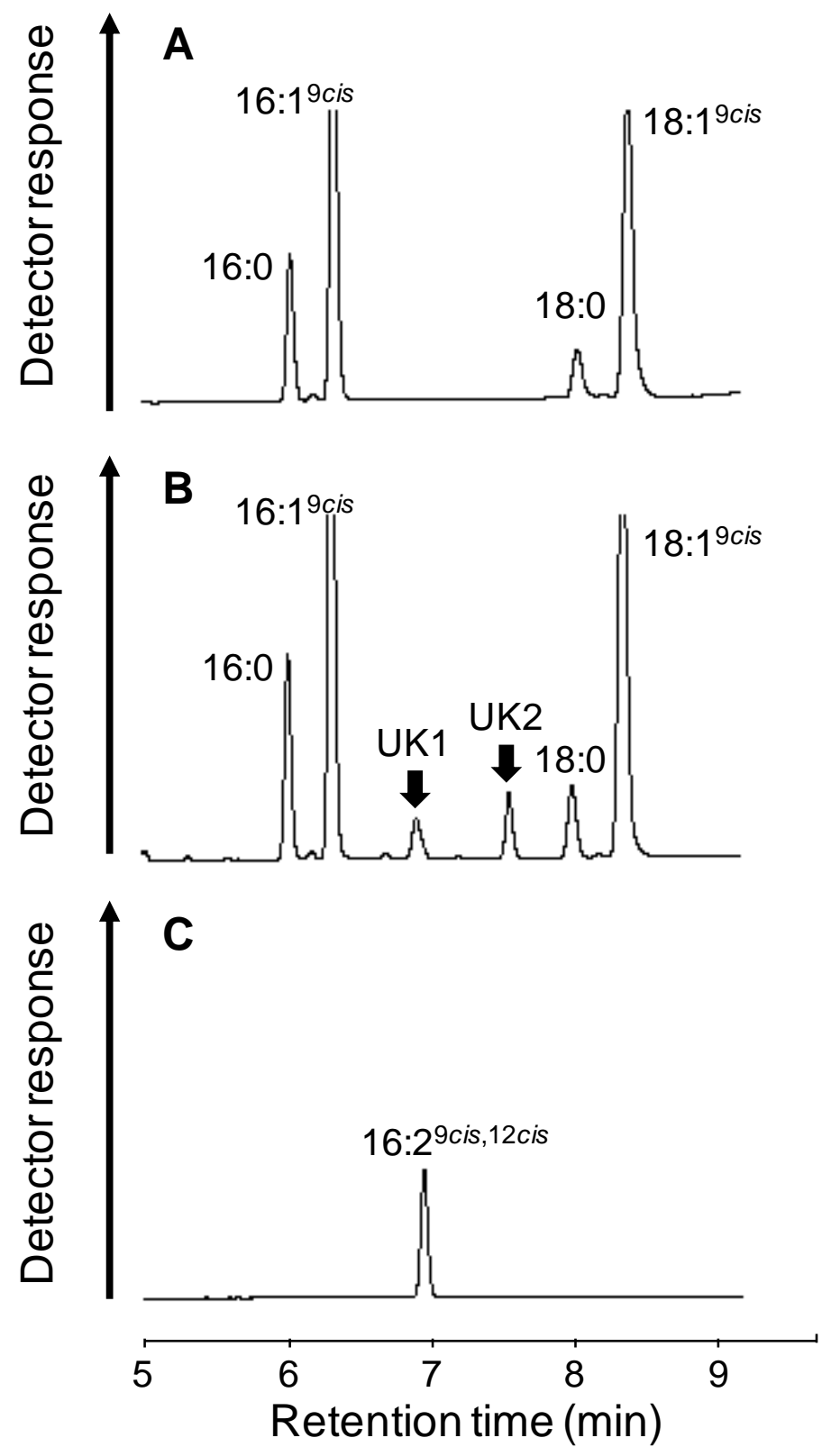

FIG.1. Kikukawa et al. 
A

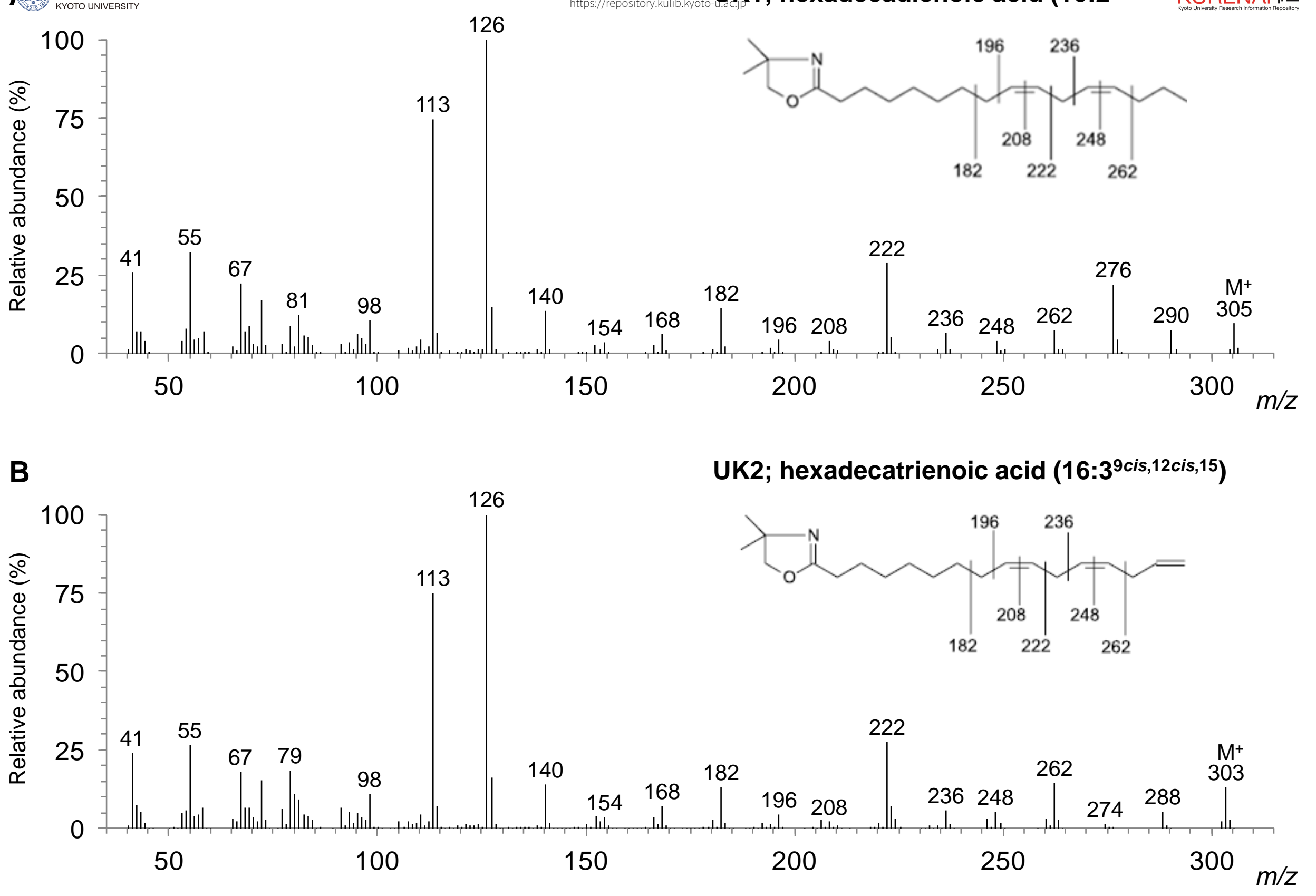

FIG.2. Kikukawa et al.

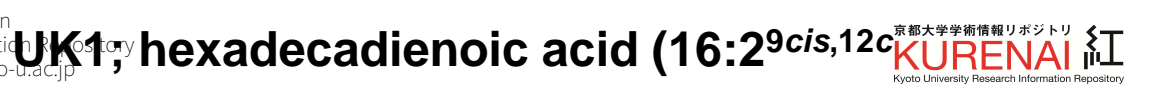

UK2; hexadecatrienoic acid (16:39cis,12cis,15)

222 

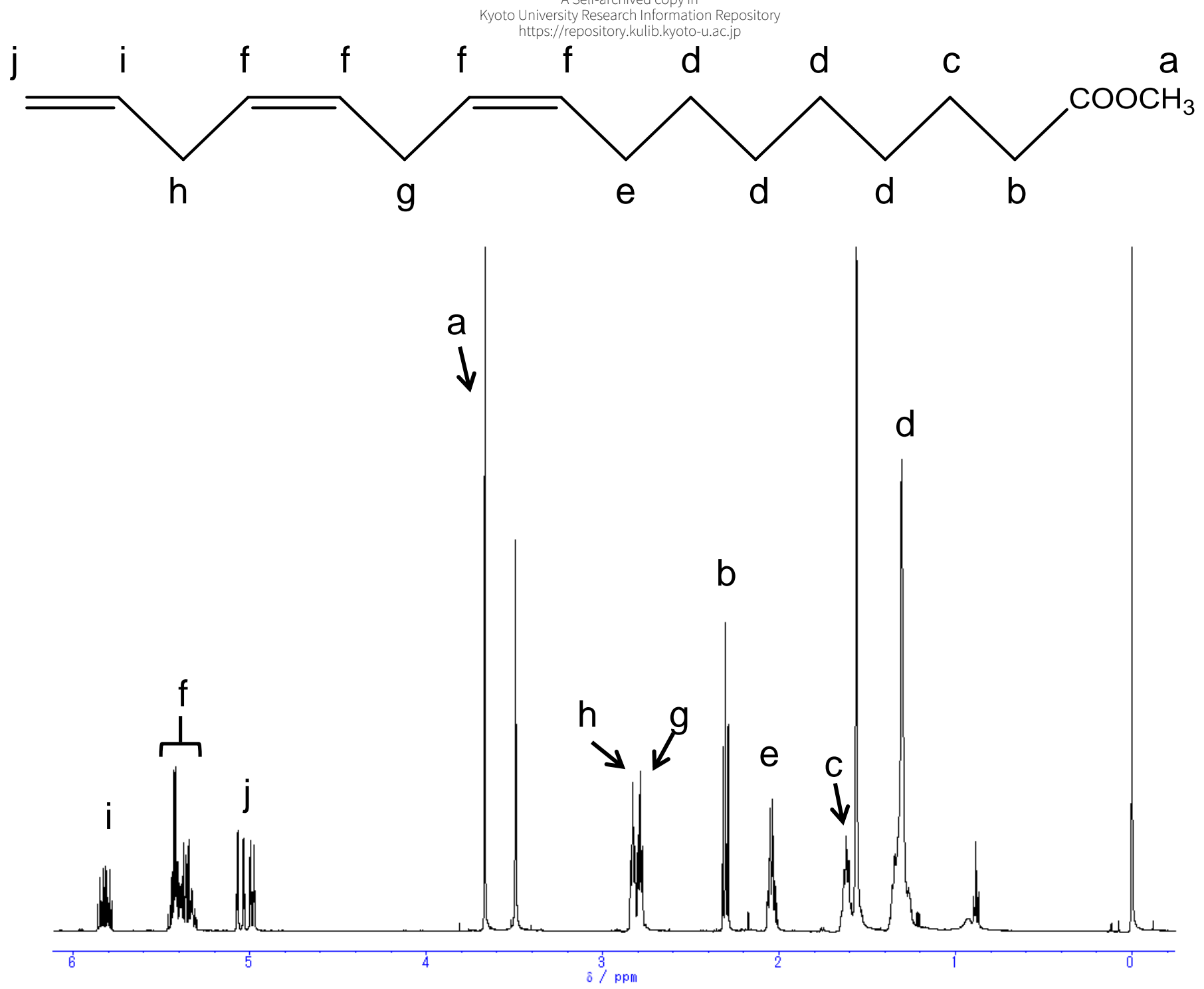

FIG. 3. Kikukawa et al. 
1 Table 1. Fatty acid compositions of the yeast transformants

2

$3 \quad$ Fatty acid

Fatty acid composition (\%)

4

Control strain maw3 transformant

5

(pYE22m) $\quad$ (pYE22MAW3)

$6 \quad 16: 0$

12.4

11.9

$7 \quad 16: 1^{9 c i s}$

45.4

40.2

$8 \quad 16: 2^{9 c i s, 12 c i s}$

N.D. ${ }^{\mathrm{a}}$

0.8

$9 \quad 16: 3^{9 c i s, 12 c i s, 15}$

N.D.

4.0

$10 \quad 18: 0$

5.7

5.2

$11 \quad 18: 1^{9 c i s}$

34.6

32.8

${ }^{a}$ N.D., not detected

13 
1 Table 2. Conversion of various fatty acids by the maw3-expressing yeast

$2 \quad$ transformant $^{\mathrm{a}}$

3

4 Substrate $\quad$ Product $\quad$ Conversion rate $(\%)^{\mathrm{b}}$

5

$15^{\circ} \mathrm{C} \quad 28^{\circ} \mathrm{C}$

6 Endogenous fatty acid

$7 \quad 16: 1^{9 c i s}$

$8 \quad 16: 2^{9 c i s, 12 c i s}$

9 Exogenous fatty acid

$10 \quad 16: 2^{9 c i s, 12 c i s}$

$11 \quad 18: 1^{9 c i s}(\mathrm{OA})$

$12 \quad 18: 1^{9 c i s, 12 y n}$

$13 \quad 18: 2^{6 c i s, 9 c i s}$

$14 \quad 18: 2^{9 c i s, 12 c i s}$ (LA)

$15 \quad 18: 2^{9 \text { cis, } 11 \text { trans }}$

$16 \quad 18: 2^{9 \text { trans, } 12 \text { cis }}$

$17 \quad 18: 2^{9 \text { trans,12trans }}$

$18 \quad 18: 2^{10 t r a n s, 12 c i s}$
$16: 2^{9 c i s, 12 c i s}$

$16: 3^{9 c i s, 12 c i s, 15}$

$16: 3^{9 c i s, 12 c i s, 15}$

N.D.

$18: 2^{9 c i s, 12 y n, 15 c i s \mathrm{~d}}$

N.D.

$18: 3^{9 c i s, 12 c i s, 15 c i s}$ (ALA)

N.D.

$18: 3^{9 \text { trans, } 12 c i s, 15 c i s \mathrm{~d}}$

N.D.

$18: 3^{10 t r a n s, 12 c i s, 15 c i s \mathrm{~d}}$
$68.8 \pm 0.6 \quad 60.3 \pm 0.6$

$11.0 \pm 0.4 \quad 19.5 \pm 1.5$

$65.2 \pm 0.6 \quad 24.4 \pm 0.4$

$63.8 \pm 0.2 \quad 40.0 \pm 3.7$

$41.3 \pm 0.2 \quad 18.1 \pm 0.5$ 
1

$18: 3^{6 c i s, 9 c i s, 12 c i s}$ (GLA)

$18: 4^{6 c i s, 9 c i s, 12 c i s, 15 c i s}(\mathrm{SDA})$

$74.8 \pm 0.1$

$32.6 \pm 0.7$

$2 \quad 20: 2^{9 c i s, 12 c i s}$

N.D.

3

$20: 3^{8 c i s, 11 c i s, 14 c i s}$ (DGLA)

$20: 4^{8 c i s, 11 c i s, 14 c i s, 17 c i s}($ ETA)

$57.7 \pm 1.1$

$6.7 \pm 0.3$

4

$20: 4^{5 c i s, 8 c i s, 11 c i s, 14 c i s}$ (ARA) $20: 5^{5 c i s, 8 c i s, 11 c i s, 14 c i s, 17 c i s}$ (EPA) $52.5 \pm 2.6$

$8.7 \pm 0.7$

5

${ }^{a}$ All exogenous free fatty acid substrates were added at $0.1 \mathrm{mM}$. All the values are for

$6 \quad$ three independent samples (mean \pm SD)

$7 \quad{ }^{\mathrm{b}}$ Conversion rate $(\%)=100 \times[$ product $] /[$ product + substrate $]$

$8 \quad{ }^{\mathrm{c}}$ N.D., not detected

$9 \quad{ }^{\mathrm{d}}$ Putative product 


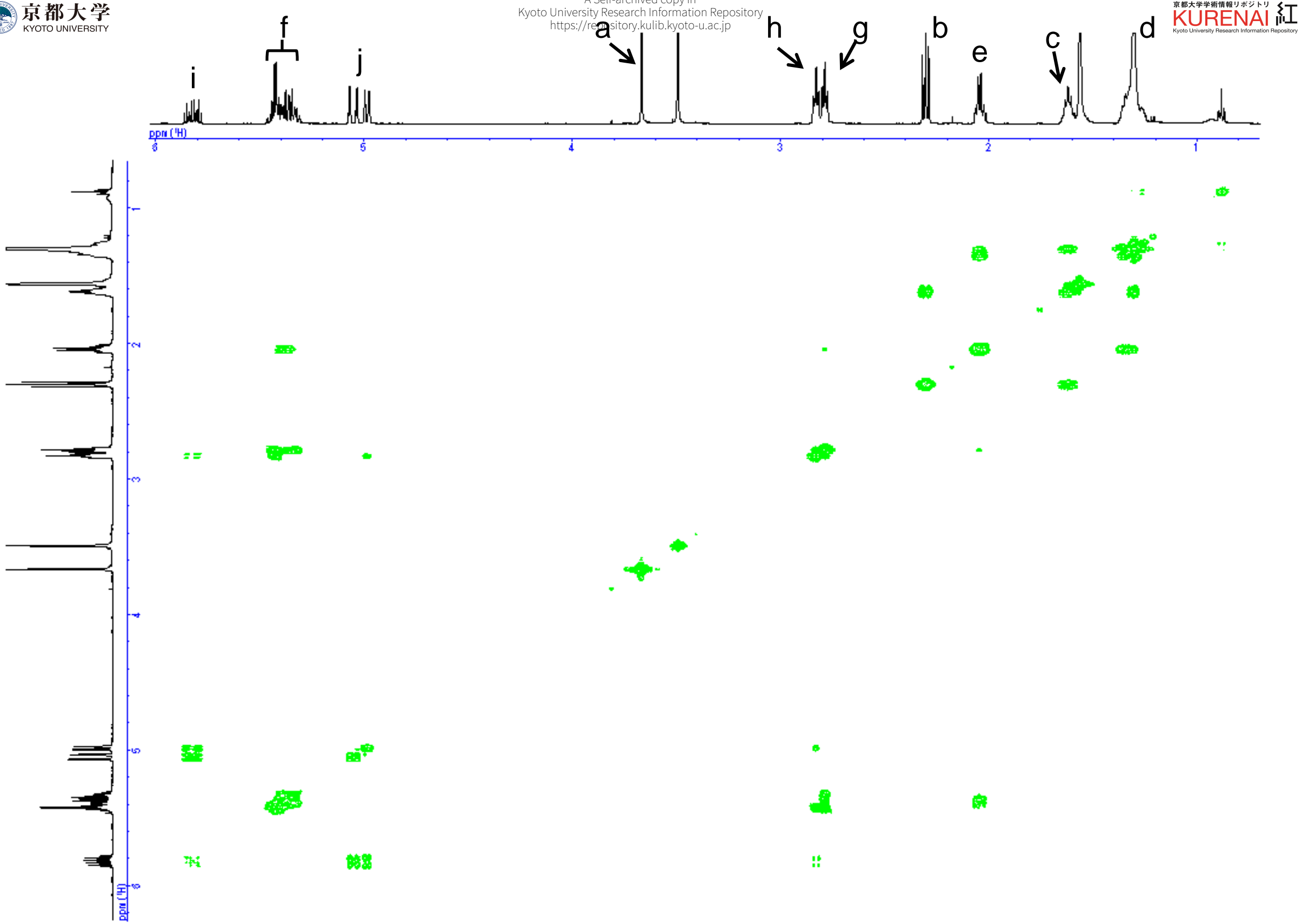

FIGURE S1. DQF-COSY analysis of the UK2 methyl ester. 